\title{
Separation quality of a geometric ratchet
}

\author{
C. Keller, Florian Marquardt, and C. Bruder \\ Departement Physik und Astronomie, Universität Basel, Klingelbergstrasse 82, CH-4056 Basel, Switzerland
}

(Received 17 December 2001; published 11 April 2002)

\begin{abstract}
We consider an experimentally relevant model of a geometric ratchet in which particles undergo drift and diffusive motion in a two-dimensional periodic array of obstacles, and which is used for the continuous separation of particles subject to different forces. The macroscopic drift velocity and diffusion tensor are calculated by a Monte Carlo simulation and by a master-equation approach, using the corresponding microscopic quantities and the shape of the obstacles as input. We define a measure of separation quality and investigate its dependence on the applied force and the shape of the obstacles.
\end{abstract}

DOI: 10.1103/PhysRevE.65.041927 PACS number(s): 87.10.+e, 05.40.-a, 02.50.Ey, 82.20.-w

\section{INTRODUCTION}

During the last decade, ratchets have been the subject of intense research efforts (for a recent comprehensive review see [1]). Ratchets are able to produce a directed current of particles although no net average force is applied. Besides the fundamental interest in such a somewhat counterintuitive physical phenomenon, their analysis is important both for the description of natural nonequilibrium transport processes (like "Brownian motors" in cells [2,3]) and for concrete technical applications as rectifiers and separation devices. The various types of ratchets considered so far include rocking, flashing, and correlation ratchets, where a temporally periodic force, periodic switching of a potential, and colored nonthermal noise, respectively, induce directed transport in an asymmetric potential $[4-6,1]$. Apart from these, there are "geometric" ratchets, which do not necessarily require any time-dependent forcing but consist, instead, of a twodimensional periodic array of asymmetric obstacles [7-12], see Fig. 1. Particles are driven by a constant external force through the array while they are undergoing diffusive motion. Because of the asymmetry of the obstacles, the particles' average drift velocity acquires a component perpendicular to the direction of the external force, which constitutes the ratchet effect. Since this is easier to realize experimentally than the time-dependent ratchets, it has been proposed and already demonstrated [10] as a device for the separation of charged biomolecules, which were subject to an external electric field and underwent diffusive motion in an array of micrometer-sized obstacles produced by a lithographic process.

In this paper, we want to take up the latter example and analyze specifically the quality of the separation effect and its dependence on various parameters. A similar numerical analysis has been carried out in Ref. [11], where the ratchet effect was investigated for a smooth periodic potential. However, we will emphasize that optimizing the ratchet effect alone is not equivalent to the optimization of the separation quality. Apart from that, we will discuss the criterion for assessment of the separation quality, point out several "trivial" possibilities for optimization and analyze the effective change in the diffusion tensor brought about by the presence of the obstacles. The discussion will center around a model of particles undergoing drift-diffusive motion on a discrete lattice. This model is analyzed numerically using both a Monte Carlo scheme as well as the numerical solutions of a master equation.

The remainder of the paper is organized as follows: First, we will review briefly the geometric ratchet used for the separation of particles, point out the distinction between microscopic and macroscopic drift velocities and diffusion tensors and establish a measure of separation quality. Then, we will set up our lattice model. Using the results of the numerical simulations, we will present the dependence of the macroscopic diffusion tensor and drift velocity on the parameter characterizing the force applied to the particles, as well as on the shape of the obstacles. Finally, we will optimize the separation quality for a situation of two particle species that are subject to different forces, for a restricted set of obstacle shapes.

\section{SEPARATION IN A GEOMETRIC RATCHET}

In a "geometric ratchet," particles drift and diffuse in a periodic potential, where the potential inside each elemen-

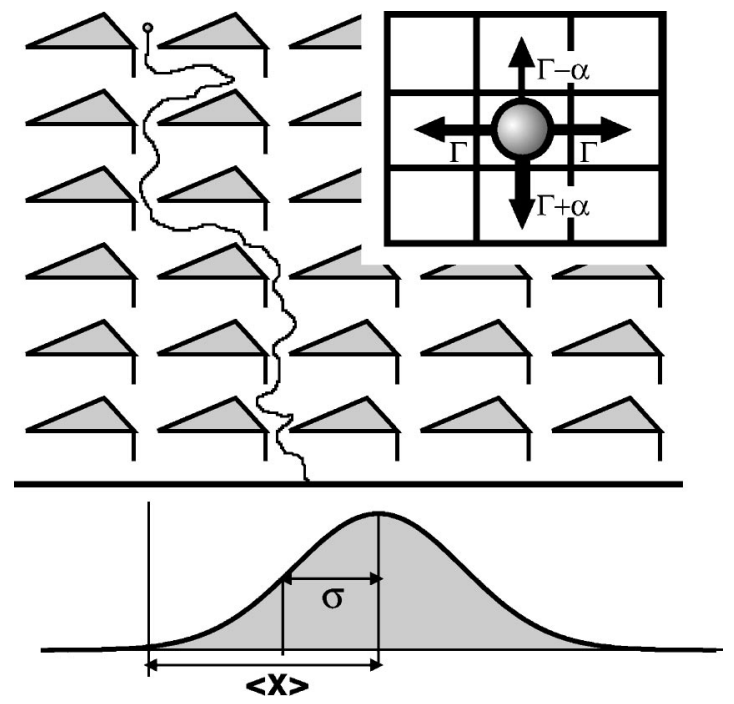

FIG. 1. The model situation: A particle diffusing and drifting through a periodic array of obstacles. When it has reached the final row of the array, its horizontal deflection is registered, which leads to a Gaussian distribution for an ensemble of many particles (after coarse graining over several obstacles). The inset shows the transition rates used in the Monte Carlo simulations on a lattice. 
tary cell is asymmetric, leading to a ratchet effect. In the present work, we will only consider the special case of a two-dimensional array of impenetrable obstacles. The drift velocity and diffusion constant of the particles are assumed to be the same everywhere outside the obstacles. While the diffusion constant is fixed, the drift velocity depends not only on the mobility but also on the force applied to the particles. If the latter derives from an electric field, it will be proportional to the charge of the particles, which is important for effecting a separation of different particle species, in the way demonstrated in Ref. [10]. In this work, differently charged biomolecules have been injected at a corner of a periodic array of obstacles. An electric field is applied to the setup so that the particles are subject to a force pointing downward. Due to the asymmetry of the obstacles, the average drift velocity has a horizontal component. Therefore, after the particles have traversed several rows of obstacles, the center of the particle distribution is deflected by a certain amount. When the magnitude of this deflection is sufficiently different for two particle species, they may be separated by collecting the particles arriving at a certain row below the injection point. This permits a continuous separation of particles, in contrast to electrophoresis. The quality of separation does not depend on the strength of the ratchet effect alone but rather on the difference in the deflections for two given species.

\section{A. Macroscopic drift and diffusion}

If the center of the particle distribution moves at a "macroscopic" average drift velocity $\overline{\vec{v}}$, then the slope of the line it traces (starting from the point of injection) is given by the ratio $\bar{v}_{x} / \bar{v}_{y}$. The average deflection in the final row is obtained by multiplying this ratio with the height of the array. Obviously, if the average drift velocity vector for one of the species is just proportional to that of the other one, no separation can result, regardless of the strength of the ratchet effect (i.e., the magnitude of the slope) itself.

Apart from the average drift, the particle distribution will also undergo diffusion, with a "macroscopic diffusion tensor" $\bar{D}$ that will be different from the microscopic one, due to the presence of the obstacles (which may hinder the expansion of the particle cloud in some directions, for example). It is important to know about the diffusive spreading of the distribution, since it affects the separation quality. A large difference in deflections for the two species will be useless if it is bought at the price of a large width of the respective distributions, which will overlap so that they cannot be separated unambiguously.

The general functional dependence of $\overline{\vec{v}}$ and $\bar{D}$ can be obtained from dimensional analysis. The microscopic parameters entering are the microscopic drift velocity $v$ (pointing along the $y$ direction), the (isotropic) diffusion constant $D$, the height of the obstacles $h$, and a collection of parameters describing their shape $S$ (including the aspect ratio). The only possibility of forming a dimensionless parameter out of a combination of $D, v$, and $h$ is given by

$$
\xi \equiv \frac{v h}{D}
$$

It is proportional to the microscopic drift velocity $v$ and, therefore, to the microscopic mobility $\mu$ multiplied by the force $F$. The parameter $\xi$ will be used to present the results of our numerical simulations of a lattice model and to compare them with the real physical parameters. The most general form of the macroscopic drift velocity and diffusion tensor is given by

$$
\begin{gathered}
\overline{\vec{v}}=v \vec{v}_{0}(\xi, S), \\
\bar{D}=D D_{0}(\xi, S) .
\end{gathered}
$$

Here, $\vec{v}_{0}$ and $D_{0}$ are dimensionless vector and tensor functions.

From these considerations, one can already conclude that two species differing only in their mobilities (but not in the forces acting on them) will not become separated, in spite of the ratchet effect. This is due to the Einstein relation $D$ $=\mu k_{B} T$, which ensures that the ratio $\mu / D$ will be the same for both species. Since the forces are assumed to be the same, $v / D$ is also unchanged, such that the respective values of $\xi$ are equal. Therefore, the average drift velocity vector only gets scaled when one passes from one species to the other, so that the drift slope remains the same, as has been discussed above.

For small values of the external force (i.e., $\xi$ ), the macroscopic drift velocity depends linearly on the force and, therefore, on $v$ (as long as the linear component is not suppressed due to symmetry). Therefore, a "macroscopic mobility tensor" $\bar{\mu}$ relating $\overline{\vec{v}}$ to the force $\vec{F}$ may be defined, such that $\overline{\vec{v}}=(\bar{\mu} / \mu) v \vec{n}$, with $\vec{n}$ being the direction of the microscopic drift. The numerical calculations (see remarks in Sec. III) will confirm that it fulfills the Onsager symmetry relations: $\bar{\mu}$ is found to be symmetric. Likewise the macroscopic diffusion and mobility tensors are connected by the Einstein relation for small values of the external force: $\bar{D}=\bar{\mu} k_{B} T$, where the temperature $T$ is obtained from the ratio $D / \mu$ of the corresponding microscopic quantities. Physically, this derives from the fact that the equilibrium distribution in a setup with a wall at the bottom is given by the Boltzmann distribution, which carries over from the microscopic density to the coarse-grained density, whose evolution is governed by $\bar{D}$ and $\bar{\mu}$. Apart from the fact that one has to be still in the regime of linear variation of $\overline{\vec{v}}$ for the Einstein relation to make sense, it can only hold as long as the force is not so strong as to make the density fall off rapidly over the scale of a single obstacle, because then the coarse-graining procedure is no longer justified. This happens approximately at $\xi \sim 1$, which is also the condition that has to be reached to see an appreciable separation effect. 


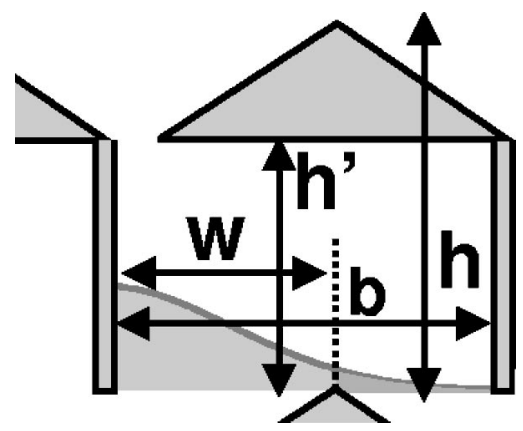

FIG. 2. Parameters entering the analytical estimate of the macroscopic drift velocity. The spreading Gaussian distribution is cut into two halves (see text).

\section{B. Analytical estimate}

The magnitude of the ratchet effect can be estimated analytically $[5,7,9]$ for high enough external force (large $\xi$ ). Then, one can treat the motion in the direction of the external force as deterministic (neglecting diffusion), so that diffusive spreading takes place only in the perpendicular direction. In this simplified picture, the geometric ratchet becomes analogous to a time-dependent one-dimensional "flashing" ratchet, with the $y$ coordinate playing the role of time. For a typical obstacle (as has been used, e.g., in the experiment of van Oudenaarden and Boxer [10]), the diffusing particle distribution (in the shape of half a Gaussian curve) will be split into two parts by the "top part" of the obstacle, see Fig. 2. Here and in the following, we assume that there is one connected obstacle. The left part proceeds downward further on, while the right part moves one cell to the right. Therefore, the slope $\bar{v}_{x} / \bar{v}_{y}$ is given essentially by the percentage of particles that have moved to the right in such an "elementary step," i.e., by an integral over the respective part of the Gaussian distribution. For large $\xi$ it can be approximated by an exponential (which becomes a good approximation if the magnitude of the following exponent exceeds 2),

$$
P(\xi) \equiv \frac{\bar{v}_{x}}{\bar{v}_{y}} \approx \frac{2 b}{\sqrt{\pi} w} \sqrt{\frac{h^{\prime}}{h}} \frac{1}{\sqrt{\xi}} \exp \left(-\frac{w^{2}}{4 h h^{\prime}} \xi\right) .
$$

Here, the dependence on $\xi$ has been made explicit, while all the other dimensionless parameters are ratios of lengths determining the shape $S$ of the obstacle (see Fig. 2). Obviously, if the force becomes very large, the particles will only move down inside "channels," since they do not have time to spread to the left or right. Then the slope $\propto \bar{v}_{x} / \bar{v}_{y}$ becomes very small, as is expressed by this formula. On the other hand, for very small forces, neglecting the possibility of diffusing backwards in the $y$ direction (or more than one cell in the $x$ direction) renders this estimate invalid. Qualitatively, however, it is correct that the slope tends to a constant in the limit of vanishing force $\xi \rightarrow 0$. Therefore, separation is ineffective both at very small forces (since the slopes of the two species are the same) and at very large forces (since the slopes differ but are both very small). Since the prefactor multiplying $\xi$ in the exponent is of the order of one, the optimum separation quality for the obstacle shape discussed here will also be reached when $\xi_{1}$ and $\xi_{2}$ are of the order of one. This is confirmed by the numerical analysis below.

\section{Quality of separation}

In the long-time limit, the ensemble of the particles of a given species (having started at the injection point) assumes the form of a two-dimensional Gaussian distribution, which drifts at a velocity $\overrightarrow{\vec{v}}$. Therefore, the distribution of particles along the $x$ coordinate of the final row is also a Gaussian, which is centered around some value $\langle x\rangle$ and has a width $\sigma$. We will assume that separation is performed by collecting all the particles up to some point $x_{0}$ in one bin and the rest in another bin. Ideally, the two bins would only receive particles of a single species ( 1 or 2 ). Due to the overlap of the two distributions, this is not possible and there is a certain percentage of particles that are attributed to the wrong bin. Qualitatively, the optimal choice of $x_{0}$ is one where this "error" is minimized. However, since there are two different types of errors (percentage of particles 1 attributed to bin 2 and vice versa), no unambiguous definition of the optimal choice of $x_{0}$ and the corresponding optimal separation quality exists. We suggest to take the separation of $\left\langle x_{1}\right\rangle$ and $\left\langle x_{2}\right\rangle$ and divide it by the maximum of the widths $\sigma_{1}$ and $\sigma_{2}$ to arrive at a measure of the separation quality, which is easily evaluated as

$$
Q \equiv\left|\left\langle x_{1}\right\rangle-\left\langle x_{2}\right\rangle\right| / \max \left(\sigma_{1}, \sigma_{2}\right) .
$$

For the parameters considered here, it seems to be appropriate.

Another possible definition consists in replacing the maximum by the geometric mean of the two widths,

$$
Q^{\prime} \equiv\left|\left\langle x_{1}\right\rangle-\left\langle x_{2}\right\rangle\right| / \sqrt{\sigma_{1} \sigma_{2}}
$$

Note, however, that there are situations when this may be a misleading measure, particularly when one of the widths $\sigma_{1}$ or $\sigma_{2}$ is much larger than the other. In these cases, $Q$ will probably be better suited.

Other measures have been used in the literature. For example, in Refs. [9,7], the authors essentially asked how large the relative difference in the diffusion constants of two species should be in order to have a separation that exceeds the spread of one of the two distributions.

In any case, any reasonably defined optimal separation quality can only be a function of two dimensionless parameters: the measure $Q$ (or $Q^{\prime}$ ) used here and the ratio $\sigma_{1} / \sigma_{2}$.

The goal is to optimize the separation quality for two given species of particles by varying the applied force and the shape of the obstacles (including their size and aspect ratio). The following parameters are naturally assumed to be fixed: the microscopic mobilities and diffusion constants of the species (connected by the Einstein relation for a given temperature), the ratio $\lambda$ of the forces (equal to the ratio of charges in an electric field), and the total height $H$ of the periodic array in the $y$ direction. The latter will be dictated by practical considerations (if it could be made arbitrarily large, one would do this to get an ideal separation effect). 
We want to argue when and why a nontrivial optimum is to be expected. It has been pointed out above that choosing the force too large or too small results in a decrease of separation quality. Likewise, one could make the ratchet effect itself arbitrarily strong, by choosing a slanted line of large slope $d x / d y$ (as one big obstacle). However, both species would drift along that line and could not be separated either.

In order to obtain a more quantitative understanding, we use the general functional forms given for $\overline{\vec{v}}$ and $\bar{D}$, and insert them into the measure $Q$ introduced above. More precisely, we use the scaling expressions $\langle x\rangle=H \bar{v}_{x} / \bar{v}_{y}$ $=H x_{0}(\xi, S)$ and $\sigma=\sqrt{D H / v} \sigma_{0}(\xi, S)$ for both species, which are assumed to have the same microscopic values of $\mu$ and $D$ but different forces acting on them, such that $v_{2}=\lambda v_{1} \equiv \lambda v$ and $\xi_{2}=\lambda \xi_{1} \equiv \lambda \xi$. Thus, we obtain

$$
Q=\frac{\left|\left\langle x_{1}\right\rangle-\left\langle x_{2}\right\rangle\right|}{\max \left(\sigma_{1}, \sigma_{2}\right)}=\sqrt{\frac{H}{h}} \frac{\sqrt{\xi}\left|x_{0}(\xi)-x_{0}(\lambda \xi)\right|}{\max \left(\sigma_{0}(\lambda \xi) / \sqrt{\lambda}, \sigma_{0}(\xi)\right)} .
$$

After the fraction in the last line has been optimized by varying both $\xi$ and the shape $S$ of the obstacles, $Q$ could be made arbitrarily large by having the height $h$ of an individual obstacle go to zero (at fixed aspect ratio), such that the array (of fixed height $H$ ) contains more rows. Since $\xi=v h / D$ must remain constant, this means that the microscopic drift velocity $v$ has to go to infinity. Physically, the enhancement of separation quality can be understood in the following way: Although the slopes will remain the same, the relative size of the diffusive spread decreases like $\sqrt{h / H}$. Note as well that due to the same reason, the maximum of the fraction does not exist, strictly speaking. It is possible to keep $h$ fixed but still have an effective increase in the number of rows by placing an array of miniaturized obstacles inside an "elementary" cell. However, in practice, there are obvious restrictions on the force that can be applied to the particles, as well as on the minimum size of the obstacles. Therefore, it is only possible to choose the force as large as possible and the corresponding value of $h$ in such a way that $\xi$ takes on the optimal value under these restrictions.

It is also helpful to consider the behavior of $Q$ for the analytical estimate given in Eq. (3), which is valid for large values of $\xi$. After $H / h$ rows (and a time $t=H / \bar{v}_{y}$ ), the average deflection is $\bar{v}_{x} t=\bar{v}_{y} P t$ and the variance of the resulting Poisson distribution is $2 \bar{D}_{x x} t=b H P$. This gives the relation $\bar{D}_{x x} / \bar{v}_{x}=b / 2$, which is confirmed by the numerical results below (for obstacles of type $A$ and $B$ in Fig. 6). We obtain

$$
Q \sim \sqrt{\frac{H}{b}} \frac{|P(\xi)-P(\lambda \xi)|}{\max (\sqrt{P(\xi)}, \sqrt{P(\lambda \xi)})} .
$$

By setting $P(\xi) \sim \exp (-\gamma \xi)$, with some exponent $\gamma$, we can find the approximate behavior of $Q$ : It drops like exp $(-\xi \gamma / 2)$ at large $\xi$, regardless of $\lambda(>1)$. On the other hand, the alternative definition $Q^{\prime}$ given above would only decrease like $\exp [-\xi \gamma(3-\lambda) / 4]$. For $\lambda>3$, this may even rise at large $\xi$, which is probably an indication that, for this regime, the expression for $Q^{\prime}$ is not any longer a good measure of separation quality: The difference in the spread of the two species grows too fast.

\section{THE LATTICE MODEL}

We have set up a model to study numerically the diffusion of particles under the influence of an external force in a periodic array of obstacles: A single particle is positioned on a point of a two-dimensional lattice, which consists of square fields. During each time step, the particle either changes its position to one of the neighboring squares (with a certain probability) or remains on its original square.

The probabilities to move to the right or to the left are both equal to $\Gamma$. In the absence of obstacles, there is consequently no net flow of particles in the horizontal direction. The probability to move downward (i.e., in the positive $y$ direction) is $\Gamma+\alpha$, the probability to move upward is $\Gamma$ $-\alpha$ (see inset of Fig. 1). The probability to remain on the original square is therefore equal to $1-4 \Gamma$. Because of the different probabilities to move upward and downward, a net flow results in the vertical direction.

The following relations hold for the microscopic drift velocity $v$ in the $y$ direction and the microscopic diffusion constant $D$ :

$$
\begin{gathered}
v=2 \alpha, \\
D=\Gamma .
\end{gathered}
$$

We assume lengths to be measured in lattice constants and time in units of the elementary time step. For the interpretation of the results, only the value of the dimensionless parameter $\xi$ is needed,

$$
\xi=v h / D=2 \alpha h / \Gamma,
$$

where $h$ is the height of an obstacle (elementary cell of the array) measured in lattice constants. From this expression, it is clear that increasing the spatial resolution $(h)$ means decreasing $\alpha / \Gamma$, such that this ratio vanishes in the physically relevant continuum limit. Actually, we have already assumed $\alpha \ll \Gamma$ in writing down Eq. (8). Otherwise, one would have to take into account that the diffusion resulting from the model stated above is anisotropic, with $D_{x x}=\Gamma$ and $D_{y y}=\Gamma-2 \alpha^{2}$. In our Monte Carlo simulation, we have usually chosen $\Gamma$ to be of the order of 0.1 and $\alpha / \Gamma$ to be less than about 0.1 .

Obstacles are represented by "forbidden" squares. If the particle would have to move onto such a square, it does not move and remains on its original square. All obstacles are arranged periodically.

Particles start in the top left square of the array. They continue moving until they have reached the final row, where their $x$ coordinate is saved. Using the results of many runs, the average deflection $\langle x\rangle$ and the standard deviation $\sigma$ of the resulting distribution can be calculated, see Fig. 1. Alternatively, a particle runs for a given number of time steps and its final coordinates are registered. In this way, the macroscopic drift velocity $\overline{\vec{v}}$ and diffusion tensor $\bar{D}$ can be obtained. 
In order to test the Monte Carlo simulation (which has been implemented in $\mathrm{C}++$ ), we have verified that the distribution of deflections in the final row is Gaussian (when coarse grained over several obstacles) and becomes independent of the precise starting position after a sufficiently large time (number of rows of the array), and all quantities show the correct scaling behavior described by Eq. (2), provided $\alpha / \Gamma$ is chosen small enough. The lattice resolution (connected with $\alpha / \Gamma$ ) has been chosen such that the results do not depend on it appreciably any more.

Furthermore, at small values of the external force (i.e., of $v$ and $\xi$ ), the macroscopic drift velocity depends linearly on this force and fulfills the Onsager symmetry relations. Likewise the Einstein relation holds: $\bar{D}=\bar{\mu} k_{B} T$.

\section{MASTER EQUATION}

While every quantity of interest (macroscopic drift velocity and diffusion tensor, average deflection, and spread of distribution) can be calculated using the Monte Carlo simulation, it is nevertheless useful to consider a master-equation solution as well. This is both because reaching a high statistical accuracy requires a large number of Monte Carlo runs and because a discussion of the master equation yields additional physical insights.

The particle distribution $p(x, y)$ is defined on the lattice, with integer coordinates $x$ and $y$. In a single time step, the distribution changes by

$$
\begin{aligned}
\delta p(x, y)= & \Gamma(p(x+1, y)+p(x-1, y))+(\Gamma-\alpha) p(x, y+1) \\
& +(\Gamma+\alpha) p(x, y-1)-4 \Gamma p(x, y)
\end{aligned}
$$

where the quantities on the right-hand side are to be evaluated at time $t$ and $\delta p \equiv p(t+1)-p(t)$. The equation shown here holds for every site $(x, y)$ that has no neighboring obstacle sites. For each "forbidden" site, the corresponding incoming and outgoing rates have to be left out. The temporal continuum limit is obtained by letting $\Gamma$ and $\alpha$ tend to zero, with their ratio kept fixed.

At large times, an ensemble of particles that has started at the injection point will be spread over many obstacle cells. Viewed on the scale of only a few cells (much less than the total spread), the distribution $p$ is periodic. Therefore, we can calculate the average drift velocity by solving for the stationary distribution $p$ defined inside the cell of a single obstacle, imposing periodic boundary conditions. $\overline{\vec{v}}$ is then given by the average velocity inside the cell, i.e., by

$$
\overrightarrow{\vec{v}}=\sum_{\text {links }} \vec{j}
$$

Here, the sum runs over all links connecting adjacent sites and the "current density" $\vec{j}$ along each link is obtained by multiplying the values of $p(x, y)$ on the two connected sites by the transition probabilities of a jump along the link, taking into account the direction of the link and leaving out blocked sites. The distribution $p$ itself is assumed to be normalized: $\Sigma p(x, y)=1$.
Actually, every site with no neighboring obstacle sites contributes just $p(x, y) \vec{v}$ to this sum, where $\vec{v}$ is the microscopic drift velocity. In this sense, the deviation of the macroscopic drift $\overline{\vec{v}}$ from $\vec{v}$ is seen to arise only from the boundaries of the obstacle. This can be understood most easily in the continuum limit, where $\vec{j}=-D \vec{\nabla} \rho+\vec{v} \rho$ ( $\rho$ being the continuous distribution). An integration by parts yields

$$
\overrightarrow{\vec{v}}=\int_{\text {cell }} \vec{j} d^{2} \vec{r}=\vec{v}+D \oint_{\text {obst. }} \rho \vec{n} d s .
$$

Here, the second integral extends over the boundary of the obstacle and $\hat{n}$ is the normal vector of that boundary (pointing away from the obstacle). Therefore, those boundaries where the density $\rho$ "piles up" (see Fig. 3 below) contribute most to the change in drift velocity. The second integral can, therefore, be interpreted as being proportional to the "force" due to the obstacle, impeding and deflecting the free flow of particles.

For the numerical solution, we first set up a (sparse) matrix corresponding to the transition rates on the lattice with periodic boundary conditions and then solve for the stationary solution $p$. This is done by setting $p(x, y)=1$ on an arbitrary nonblocked site and striking out the respective column and row in the homogeneous linear system of equations, such that it becomes inhomogeneous and nonsingular. We have used a sparse matrix bi-conjugate-gradient solver from the LAPACK package of linear algebra routines [13]. For a typical cell size of $128 \times 128$, the number of nonvanishing matrix entries is about $10^{5}$. In the end, $p$ is normalized and the current density and macroscopic drift velocity are calculated. This type of calculation has already been performed by the authors of Ref. [11] for a model with a smoothly varying periodic potential.

It is also possible to calculate the macroscopic diffusion tensor $\bar{D}$ using the master equation. This involves the solution of an inhomogeneous linear equation, with the linear operator defined by Eq. (10) and the inhomogeneity derived from the solution $p$ of the homogeneous equation. The derivation of the equation and the formula for $\bar{D}$ can be found in the Appendix. To the best of our knowledge, such an analysis has not been carried out before.

\section{NUMERICAL RESULTS}

In this section, we present the results of both the Monte Carlo simulations and solutions of the master equation.

In the Monte Carlo simulation, the relative statistical accuracy of the macroscopic drift velocity $\left(\delta \bar{v}_{x} / \bar{v}_{x}\right.$, etc.) is given approximately by $\sqrt{D /\left(v^{2} N t\right)}$, while that of $\bar{D}$ is estimated to be $1 / \sqrt{N}$, where $N$ is the number of samples and $t$ is the number of time steps. Independence of the detailed initial conditions is reached when the diffusive spread $\sqrt{D t}$ becomes much larger than the width of an obstacle cell (typically 160). We have chosen values of $t \geqslant 10^{7}$ and $N \geqslant 10^{3}$ in order to fulfill these criteria. 

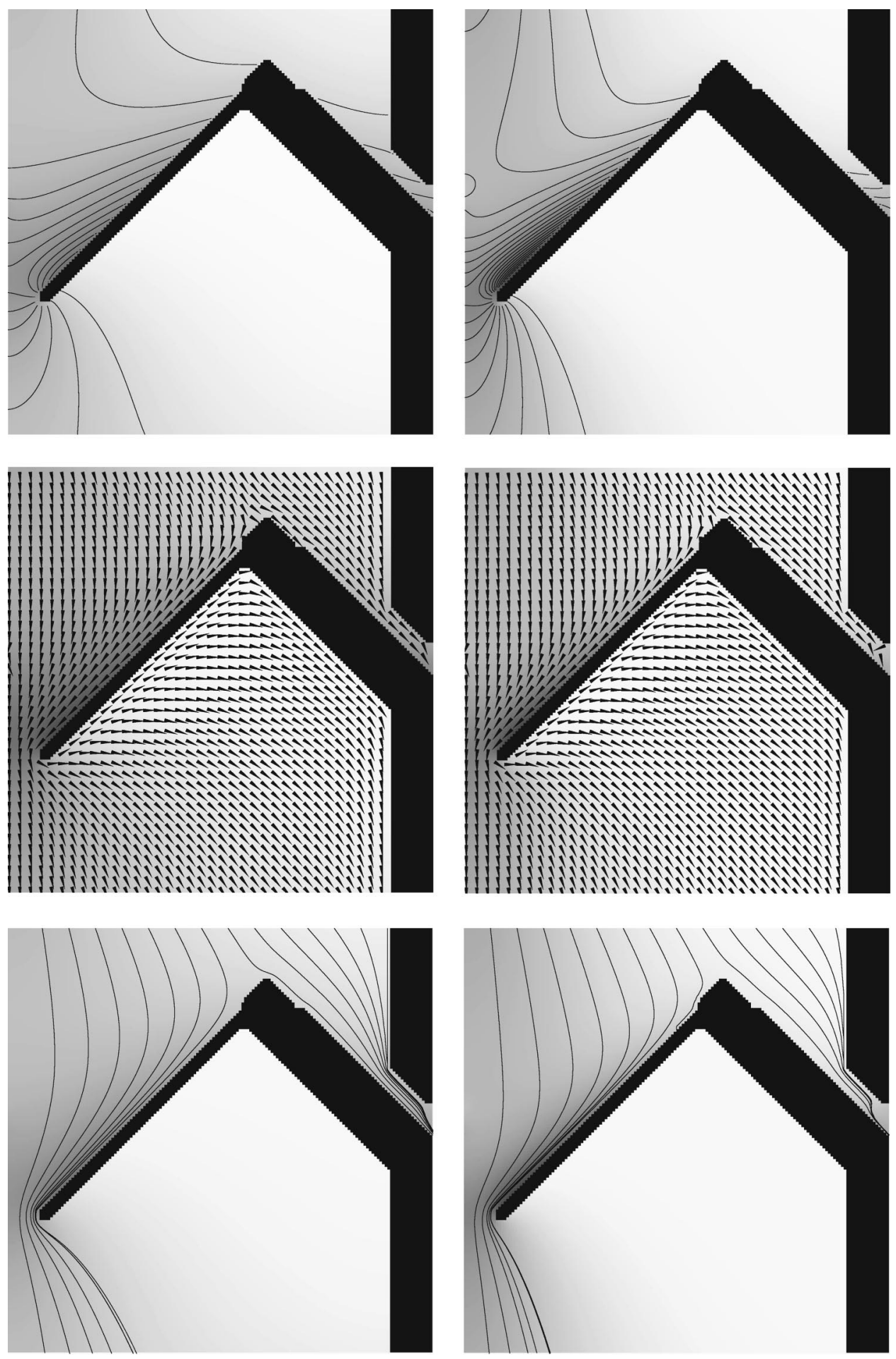

FIG. 3. Distribution $p$ (top), velocity direction field $\hat{v}$ (middle), and "streamlines" (bottom) for low and high force (left and right columns). The strength of the force $\xi=12$ for the left column and $\xi=24$ for the right column, with the microscopic drift pointing downward. Darker shading signifies increased $p$. Contour lines for $p$ have been chosen at the same (equidistant) values for the two pictures at the top. Note that the streamlines serve to visualize the direction of the current density, while the real motion of a single particle is governed by drift and diffusion. The "velocity direction" is the normalized current density vector field. Note the stronger pileup in density at the left "roof" of the obstacle for the stronger force, as well as the distortion of streamlines there and in the narrow channel at the right of the pictures. The lattice shown here consists of $160 \times 160$ sites.

\section{A. Density distribution and flow field}

The particle distribution $p$ and corresponding current density $\vec{j}$ resulting from the solution of the master equation for a typical obstacle shape are depicted in Fig. 3, both for a low and a high value of $\xi$. This corresponds to the optimal situation for the separation of two species, where one of them is almost not deflected at all (high force, i.e., high $\xi$ ), while the other one has an appreciable probability to go to a neighboring cell to the right, due to diffusion around the top part of the obstacle.

\section{B. Macroscopic drift velocity and diffusion tensor}

The results for the components of the macroscopic drift velocity are displayed in Fig. 4. These data have been obtained for the obstacle of Fig. 3, using both the master equation with periodic boundary conditions and the Monte Carlo simulation. As has been explained in a preceding section, the onset of a nonlinear dependence of $\bar{v}_{y}$ and $\bar{v}_{x}$ on $\xi$ at rather low $\xi$ implies that the obstacle is well suited for obtaining a separation effect (rather than merely a ratchet effect). 


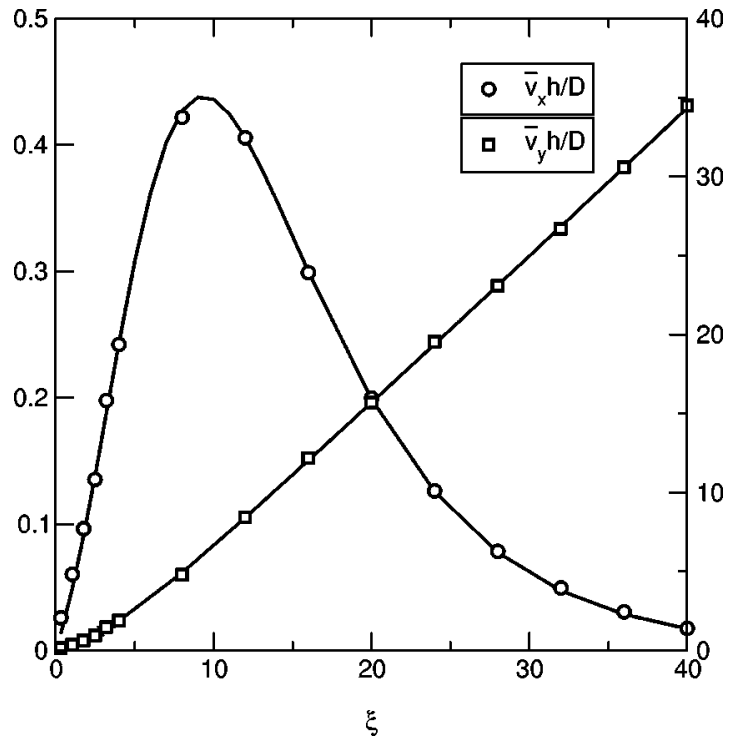

FIG. 4. Macroscopic drift velocity components $\bar{v}_{x}$ (circles, left scale) and $\bar{v}_{y}$ (squares, right scale) in units of $D / h$, plotted vs $\xi$ $=v h / D$, for the obstacle shape shown in Fig. 3, with a lattice resolution of $160 \times 160$. The lines indicate the results of the master equation, the symbols (which are larger than the error bars) are from Monte Carlo simulations $\left(t=2 \times 10^{7}, N=10^{3}\right)$.

In Fig. 5, the components of the (symmetrized) macroscopic diffusion tensor $\bar{D}$ have been plotted vs $\xi$ for the same obstacle. At low values of the driving force, all components of $\bar{D}$ are generally reduced compared with the microscopic diffusion constant $D$, since the obstacles hinder the free dif-

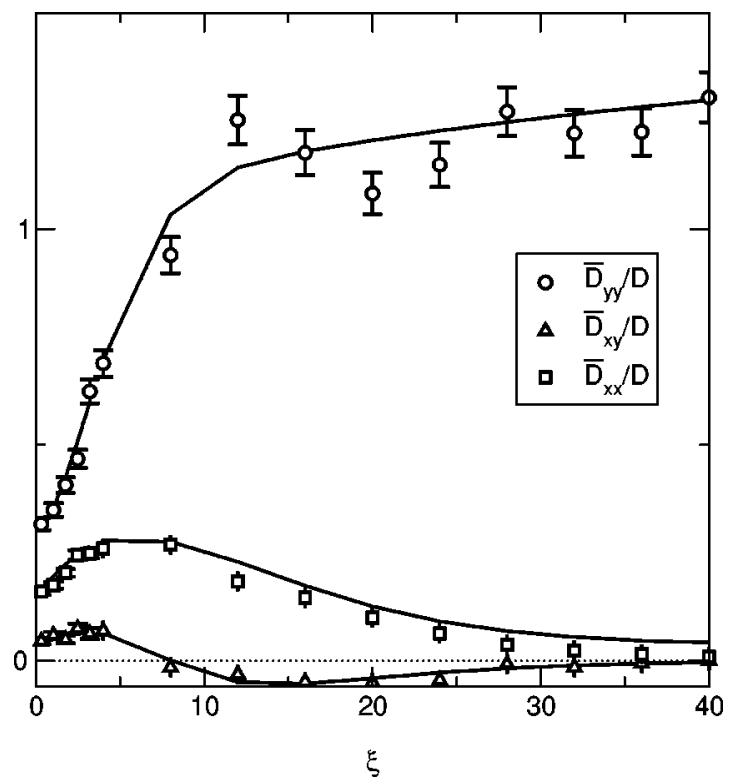

FIG. 5. Components of macroscopic diffusion tensor vs $\xi$ for the obstacle of Fig. 3. Circles, $\bar{D}_{y y} / D$; squares, $\bar{D}_{x x} / D$; triangles, $\bar{D}_{x y} / D$. Symbols show results of the Monte Carlo simulation, lines show those of the master equation. We attribute the deviation at higher values of $\xi$ (and, therefore, $\alpha / \Gamma$ ) to effects of the finite grid resolution. fusion of particles. At higher forces, the $y$ component is increased, while the $x$ component $\bar{D}_{x x}$ is further reduced. This can be understood in the following way: The motion proceeds inside vertical "channels," such that the particle cannot move easily in the horizontal $(x)$ direction, while the diffusion in the $y$ direction is more or less free. Comparing the data for $\bar{D}_{x x}$ and $\bar{v}_{x}$ shows that $\bar{D}_{x x} / \bar{v}_{x} \approx b / 2$ at larger values of $\xi$ [to the right of the maximum of $\bar{v}_{x}(\xi)$ ], as expected: both quantities decrease exponentially. Note that the off-diagonal component $\bar{D}_{x y}$ of the macroscopic diffusion tensor changes sign at about $\xi \approx 8$, which seems to approximately coincide with the sign change in $\vec{\nabla} \bar{v}_{x} / \vec{\nabla} \bar{v}_{y}$ (see Fig. 4). We have not come up, however, with an explanation for the approximate correlation between $\bar{D}_{x y} / D$ and $\vec{\nabla} \bar{v}_{x} / \vec{\nabla} \bar{v}_{y}$ yet.

The statistical accuracy of the Monte Carlo results for $\bar{D}$ is worse than that of the $\bar{v}$ results, as expected. The deviation between the results of the master equation and Monte Carlo simulation at larger values of $\xi$ is reduced when the grid resolution is enhanced (i.e., when $\alpha / \Gamma$ gets smaller for fixed $\xi)$. In the examples shown here, $\alpha / \Gamma$ takes on a maximum value of about 0.1 .

\section{Optimization of separation quality}

For the obstacle discussed above, the slope $\bar{v}_{x} / \bar{v}_{y}$ (proportional to the average deflection $\langle x\rangle$ in the last row) is shown in Fig. 6, together with those of other obstacle shapes discussed further below. The exponential decay is consistent with the analytical estimate derived in Sec. II B, see Eq. (3). From the slope of the logarithmic plot, a value of about 0.17 has been obtained for the prefactor $w^{2} /\left(4 h h^{\prime}\right)$ in the exponent of Eq. (3), which is roughly consistent with the geometrical parameters of the obstacle. Given the slope and the spread $\sigma$ (derived from the components of $\bar{D}$ ), one can obtain the separation quality $Q$ defined in Eq. (5), if one assumes some ratio $\lambda=\xi_{2} / \xi_{1}$ of the forces acting on the two species (see Fig. 7).

\section{Influence of the obstacle shape}

Both the magnitude of the ratchet effect and the separation quality depend very much on the shape of the obstacle. We have not performed a systematic search over obstacle shapes for a kind of "global" optimization of the separation quality because of the numerical effort involved. However, there are a few general properties resulting from certain geometrical features. These are illustrated by the numerical results for the slope $\bar{v}_{x} / \bar{v}_{y}$ and the separation quality $Q$ plotted in Figs. 6 and 7. They can be summarized as follows.

The vertical "wall" at one side of the obstacle $A$ depicted in Fig. 3 acts to prevent particles from diffusing back to the left, thereby increasing $\bar{v}_{x}$ and leading to a better ratchet and separation effect. This can be seen by comparing against a version with a shorter wall $(B)$. The triangular "roof" of the obstacle splits the particle distribution into two halves as it drifts downwards. If the external force is high, the particles 

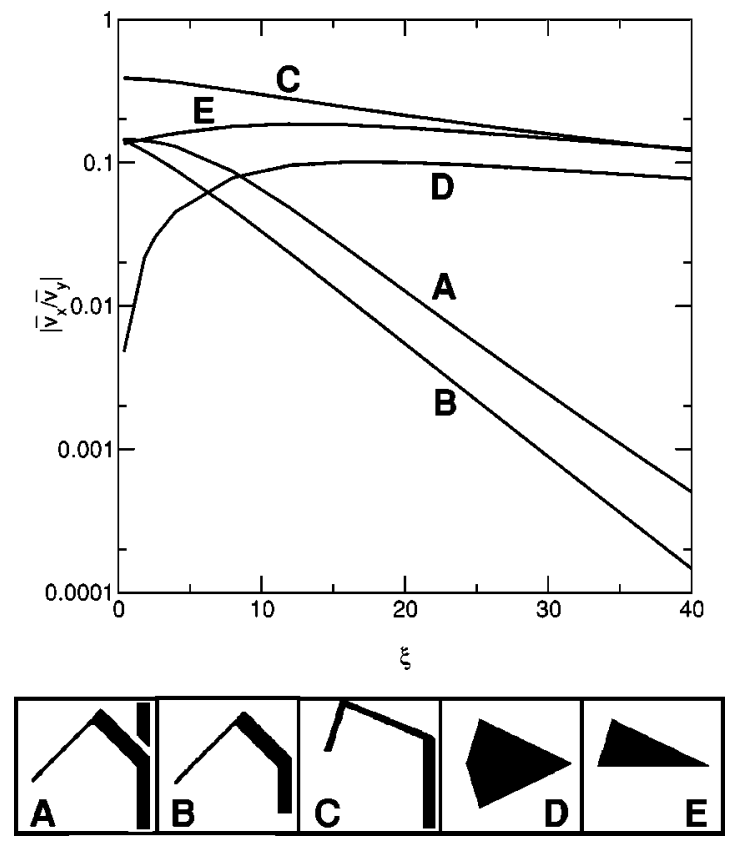

FIG. 6. Slope $\langle x\rangle / H=\bar{v}_{x} / \bar{v}_{y}$ plotted vs $\xi$. The obstacle shapes are shown at the bottom of the plot and are discussed in the text. $A$ refers to the obstacle shape shown in Fig. 3.

do not have time to diffuse a sufficient distance to the right and will be deflected back by the left side of this "roof," therefore streaming downward, with no net deflection to the right. The horizontal position of the upper tip determines the strength of the force where this transition takes place: If it is moved to the left $(C)$, a much higher force is necessary. In that case, the slope $\bar{v}_{x} / \bar{v}_{y}$ falls off more slowly with increasing force $\xi$. At the same time, the value of the slope is generally increased at small forces, since more particles are deflected one cell to the right.

In order to illustrate the difference between having a strong ratchet effect and a good separation effect, we have tried a triangular obstacle $(E)$, which yields a comparatively large slope that, however, does not change very strongly with applied force. By flipping the triangle along the horizontal axis, an obstacle with reflection symmetry is created $(D)$. This has the peculiar feature $[11,12]$ that, for symmetry reasons, $\bar{v}_{x}$ is an even function of the microscopic velocity $v_{y}$ (driving force $\xi$ ), so the linear mobility at low driving force vanishes. In principle, this nonlinear dependence of $\bar{v}_{x}$ on $\xi$ is well suited for achieving a separation effect. However, it must be kept in mind that at low values of the external force the diffusive motion is dominant, so the spread and the overlap of the particle distributions of the two species in the final row is significant. Therefore, the separation quality decreases towards $\xi=0$.

The separation quality can become zero for a special value of the external force whenever the slope shown in Fig. 6 has an extremum as a function of microscopic velocity $\xi$, such that two different $\xi$ can produce the same slope. This occurs for two of the obstacles ( $D$ and $E$ in Fig. 7).

For most of the obstacle shapes considered here, there is at most a local maximum of the separation quality at low

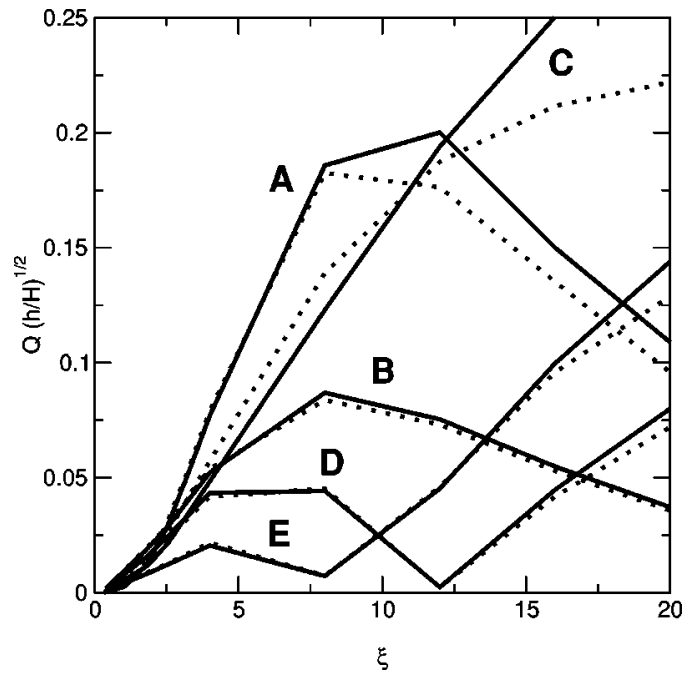

FIG. 7. Normalized separation quality $Q \sqrt{h / H}$ vs $\xi$, where $\xi$ corresponds to the force on the first species and $\xi_{2}=\lambda \xi$, with $\lambda$ $=2$ in this plot. Full lines, results of Monte Carlo simulation; dotted lines: Master equation. The difference is due to the larger values for the diffusion tensor component $\bar{D}_{x x}$ at high force $\xi$ yielded by the master equation. The location of the optimum does not change much. See discussion in the text.

forces. The global maximum is expected to occur at much higher values of the force, which may be unattainable in the experiment (and are difficult to reach in a numerical simulation with a finite lattice resolution). However, for a wellsuited obstacle like the one depicted in Fig. 3, the quality peaks at moderate values of the force.

\section{CONCLUSIONS}

In this work, we have analyzed a geometric ratchet consisting of a two-dimensional array of obstacles, where particles perform drift-diffusive motion under the action of a constant external force. We have carried out numerical calculations using both a Monte Carlo simulation and a masterequation solution in order to obtain the dependence of the "macroscopic" drift velocity and diffusion tensor on external force and obstacle shape. Using these results, we have quantified the quality of the separation effect that can be achieved when two species of particles with differing microscopic mobilities are injected into the array. Our results show the strong dependence on several features of the shape of the obstacles and demonstrate the distinction between a strong ratchet effect and a good separation effect.

\section{ACKNOWLEDGMENT}

We thank Hanno Gassmann for helpful discussions.

\section{APPENDIX: CALCULATING THE MACROSCOPIC DIFFUSION TENSOR USING THE MASTER EQUATION}

Solving the master equation for the probability density inside a cell containing a single obstacle allows one to obtain easily a numerically exact result for the macroscopic drift 
velocity $\overline{\vec{v}}$. It is given by the average flow velocity of particles inside the cell, i.e., the integral (or sum) over the current density [see Eqs. (11) and (12)]. However, to assess the quality of separation of a given geometric ratchet, it is equally important to know the macroscopic diffusion tensor, which governs the spreading of the macroscopic particle density (i.e., the density averaged over many obstacles). Its evaluation using the Monte Carlo simulation requires a large number of samples, to obtain a good statistical accuracy. Therefore, it is desirable to calculate $\bar{D}$ using the master equation as well. The steps involved in the derivation of $\bar{D}$ are slightly more involved than the straightforward calculation of $\overline{\vec{v}}$.

Our strategy is to derive the equation of motion for the macroscopic density $\bar{\rho}$,

$$
\partial_{t} \bar{\rho}=\bar{D}_{i j} \partial_{i} \partial_{j} \bar{\rho}-\overline{\vec{v}} \vec{\nabla} \bar{\rho},
$$

from the analogous equation for the microscopic density $\rho$, making use of the slow variation of $\bar{\rho}$.

If the particle density $\rho$ is spread over many obstacle cells (as is the case after waiting for a sufficiently long time), it may, in a first approximation, be described by

$$
\rho=\rho_{0} \bar{\rho},
$$

where $\rho_{0}$ refers to the detailed density that varies on the scale of a single obstacle but is periodic throughout the array. $\rho_{0}$ has been obtained before by solving the master equation for a single cell, using periodic boundary conditions at the borders of the cell and the restriction for the current density $\vec{j}_{0}$ to run parallel to the walls of the obstacle. If $\bar{\rho}$ were constant, this would constitute a (not normalizable) stationary periodic solution to the Fokker-Planck equation. However, $\bar{\rho}$ is assumed to vary (very slowly), such that this is not a stationary solution and does not fulfill the boundary conditions exactly. Therefore, it has to be supplemented by "correction terms," which depend (necessarily linearly) on the spatial derivatives of $\bar{\rho}$. Consequently, we take $\rho$ to be given by

$$
\rho=\rho_{0} \bar{\rho}+g^{i} \partial_{i} \bar{\rho}+K^{i j} \partial_{i} \partial_{j} \bar{\rho}+\cdots .
$$

Here, $g^{i}$ and $K^{i j}$ are as yet unknown periodic functions (that vary on the scale of a single obstacle). We emphasize that, of course, many different microscopic densities $\rho$ yield the same macroscopic density $\bar{\rho}$. Therefore, $\rho$ is not uniquely specified if $\bar{\rho}$ is given. However, in the long-time limit assumed here, $\rho$ has "equilibrated" and the deviations from $\rho_{0}$ are in a one-to-one correspondence with the lowest order spatial derivatives of $\bar{\rho}$.

Our further strategy is as follows: We will first rederive the known result for the macroscopic drift velocity $\overline{\vec{v}}$, which is the constant coefficient appearing in the part of the equation of motion for $\bar{\rho}$ that contains the spatial derivatives of first order: $\partial_{t} \bar{\rho}=-\overline{\vec{v}} \vec{\nabla} \bar{\rho}$. To this end, we will insert the ansatz (A3) into the equation of motion for $\rho$, keeping only the terms containing up to first derivatives of $\bar{\rho}$ and eliminating $g^{i}$ using the boundary condition. Using the result for $\overline{\vec{v}}$, we will obtain an inhomogeneous linear equation for $g^{i}$ itself, which must be solved numerically. The relation between $g^{i}$ and the macroscopic diffusion tensor $\bar{D}$ will be obtained by going to the second order in the spatial derivatives of $\bar{\rho}$. Although this involves the unknown function $K^{i j}$, we will be able to eliminate it in the same way that $g^{i}$ had been eliminated in the first step.

Let us first derive a boundary condition for $g^{i}$. The current densities for the first and second term on the right-hand side of Eq. (A3) are given by

$$
\begin{gathered}
\vec{j}=\vec{j}_{0} \bar{\rho}-D \rho_{0}(\vec{\nabla} \bar{\rho}), \\
\left(\vec{j}_{1}\right)_{l}=-D\left(\partial_{l} g^{i}\right)\left(\partial_{i} \bar{\rho}\right)+\vec{v}_{l} g^{i}\left(\partial_{i} \bar{\rho}\right)-D g^{i} \partial_{l} \partial_{i} \bar{\rho} .
\end{gathered}
$$

Here, $\vec{j}_{0}=(-D \vec{\nabla}+\vec{v}) \rho_{0}$ is the current density of $\rho_{0}$ alone. We demand $\vec{j}+\vec{j}_{1}$ to be parallel to the obstacle wall, keeping only terms including first order derivatives of $\bar{\rho}$ and then canceling these terms. This leads to the following boundary condition for $g^{i}$ :

$$
\hat{n} \vec{j}_{g i}=D \hat{n}_{i} \rho_{0},
$$

where $\vec{j}_{g^{i}}=(-D \vec{\nabla}+\vec{v}) g^{i}$ is the current density related to $g^{i}$ and $\hat{n}$ is the outer normal vector of the obstacle wall.

In the next step, we calculate $\partial_{t} \rho=-\vec{\nabla}\left(\vec{j}+\vec{j}_{1}+\cdots\right)$ up to first order in the spatial derivatives of $\bar{\rho}$ and demand $\rho_{0} \partial_{t} \bar{\rho}$ $\approx-\rho_{0} \overline{\vec{v}} \vec{\nabla} \bar{\rho}$, which is essentially the drift term for $\bar{\rho}$. Physically, this equation means that a nonvanishing slope of $\bar{\rho}$ will lead to an overall increase (or decrease) of the microscopic density $\rho$ inside an obstacle cell. The detailed shape of the distribution within that cell is not changed, only its magnitude. After dropping the overall factor $\partial_{i} \bar{\rho}$, we arrive at

$$
\vec{\nabla} \vec{j}_{g}=-2\left(\vec{j}_{0}\right)_{i}+\left(\vec{v}_{i}+\overline{\vec{v}}_{i}\right) \rho_{0} .
$$

It is not necessary to know $g^{i}$ in order to obtain $\overline{\vec{v}}$. We integrate both sides of this equation over the whole cell, assuming periodic boundary conditions for $g^{i}$ (as well as for $\left.\rho_{0}\right)$. The boundary term resulting from the walls of the obstacle contains $g^{i}$, but it can be transformed using Eq. (A5), such that we end up with an equation where $g^{i}$ has been eliminated,

$$
-D \oint \hat{n}_{i} \rho_{0} d s=-2 \int\left(\vec{j}_{0}\right)_{i} d^{2} \vec{r}+\left(\vec{v}_{i}+\overrightarrow{\vec{v}}_{i}\right) .
$$

The integral on the left-hand side runs along the obstacle wall, while that on the right-hand side extends over the whole cell. Since

$$
\int\left(\vec{j}_{0}\right)_{i} d^{2} \vec{r}=\vec{v}_{i}+D \oint \hat{n}_{i} \rho_{0} d s,
$$

we have

$$
\overrightarrow{\vec{v}}_{i}=\int\left(\vec{j}_{0}\right)_{i} d^{2} \vec{r}
$$


as has been assumed in the main text already [see Eqs. (11) and (12)].

Inserting $\overrightarrow{\vec{v}}$ into Eq. (A6) yields an inhomogeneous linear partial differential equation for $g^{i}$ which has to be solved numerically [assuming periodicity and the boundary condition Eq. (A5)]. Note that $g^{i}$ is only determined up to a constant multiple of $\rho_{0}$, since $\rho_{0}$ solves the homogeneous equation. However, as we will see, this does not affect the result for the diffusion tensor $\bar{D}$ to be derived from $g^{i}$. Further remarks concerning the numerical solution of the master equation on the discrete lattice can be found at the end of this appendix.

The current density related to the part of $\rho$ that involves second derivatives of $\bar{\rho}$ [see Eq. (A3)] is equal to

$$
\left(\vec{j}_{2}\right)_{l}=\left(\partial_{i} \partial_{j} \bar{\rho}\right)\left[\left(-D \vec{\nabla}_{l}+\vec{v}_{l}\right) K^{i j}\right]+\cdots,
$$

where we have neglected higher derivatives of $\bar{\rho}$. We arrive at a boundary condition for $K^{i j}$ at the walls of the obstacle in the same way as for $g^{i}$, by demanding $\vec{j}_{t o t} \equiv \vec{j}+\vec{j}_{1}+\vec{j}_{2}$ to be parallel to the wall. This time, we keep only the terms including second derivatives of $\bar{\rho}$. This leads to

$$
[\hat{n}(-D \vec{\nabla}+\vec{v})] K^{i j}=D \hat{n}_{j} g^{i} .
$$

To this order, the time derivative of $\rho, \partial_{t} \rho=-\vec{\nabla} \vec{j}_{t o t}$, includes both the diffusion of $\bar{\rho}$ and the drift of the term $g^{i} \partial_{i} \bar{\rho}$,

$$
\vec{\nabla} \vec{j}_{t o t}=\overline{\vec{v}}_{l} g^{i} \partial_{l} \partial_{i} \bar{\rho}-\rho_{0} \bar{D}_{l i} \partial_{l} \partial_{i} \bar{\rho}+\cdots,
$$

keeping only the second order with respect to the spatial derivatives of $\bar{\rho}$ on both sides of the equation.

As before, we integrate this equation over the cell and use the boundary condition Eq. (A11) at the obstacle walls to eliminate $K^{i j}$. The resulting expression for $\bar{D}$ then is given by

$$
\bar{D}_{j i}=D\left(\delta_{j i}-\oint \hat{n}_{j} g^{i} d s\right)+\left(\overline{\vec{v}}_{j}-\vec{v}_{j}\right) \int g^{i} d^{2} \vec{r}
$$

Note that adding $\lambda \rho_{0}$ to $g^{i}$ (with an arbitrary constant $\lambda$ ) does not affect the result for $\bar{D}$, due to Eqs. (A8) and (A9).
For the numerical solution, it is, in principle, possible to discretize Eqs. (A5) and (A6) for $g^{i}$ as well as the expression (A13) for $\bar{D}$. However, this is guaranteed to coincide with the results of the Monte Carlo simulation only in the continuum limit (where, e.g., $\alpha / \Gamma \rightarrow 0$ ). In order to have a better agreement even when one is not yet in the continuum limit, it is advisable to start directly from the discretized master equation and redo the steps of the derivation shown here for the discrete lattice.

The equation that has actually been solved numerically to arrive at $g^{i}$ is given by

$$
L g^{i}=-p \overline{\overrightarrow{v_{i}}}-\Gamma\left(p^{i+}-p^{i-}\right)-\delta_{i 2} \alpha\left(p^{i+}+p^{i-}\right) .
$$

Here, $L g^{i}$ corresponds to $-\vec{\nabla} \vec{j}_{g}$. Therefore, $L$ is the matrix kernel, which is also used for solving the homogeneous equation, $L p=0$, including the same treatment of obstacle walls and periodic boundary conditions [see the right-hand side of Eq. (10) for the definition of $L]$. $p$ is evaluated at the "current site" (the site that the left-hand side refers to), while $p^{i+}$ and $p^{i-}$ are evaluated at the neighboring sites, in positive or negative directions $i$ ( $=1,2$, corresponding to $x, y)$, respectively. At the obstacle walls, these neighboring sites may turn out to be "forbidden," in which case $p^{i+}$ or $p^{i-}$ vanishes. This implements the discrete version of the boundary condition discussed above. (Note that according to the convention used here, the microscopic drift velocity is assumed to point in the negative $y$ direction if $\alpha$ is positive).

In order to evaluate $\bar{D}$, we must carry out a sum over all sites at the wall of the obstacle, i.e., those that have forbidden sites as neighbors. This sum is denoted by $\Sigma_{W}$. The sum extending over all allowed sites in the cell is denoted by $\Sigma$,

$$
\bar{D}_{j i}=\Gamma\left(\delta_{j i}-\sum_{W} \hat{n}_{j} g^{i}\right)+\left(\overline{\vec{v}}_{j}-\vec{v}_{j}\right) \sum g^{i}-\alpha \delta_{j 2} \sum_{W} g^{i} .
$$

The last term vanishes in the continuum limit but is important to ensure that $\bar{D}$ does not change on adding a homogeneous solution $\lambda p$ to $g^{i}$.
[1] P. Reimann, Phys. Rep. 361, 57 (2002).

[2] R. D. Astumian and M. Bier, Phys. Rev. Lett. 72, 1766 (1994).

[3] R. D. Astumian, Science 276, 917 (1997).

[4] J. F. Wambaugh et al., Phys. Rev. Lett. 83, 5106 (1999).

[5] L. P. Faucheux, L. S. Bourdieu, P. D. Kaplan, and A. J. Libchaber, Phys. Rev. Lett. 74, 1504 (1995).

[6] P. Hänggi and R. Bartussek, in Nonlinear Physics of Complex Systems - Current Status and Future Trends, edited by J. Parisi, S. C. Müller, and W. Zimmermann, Springer Lecture Notes in Physics Vol. 476 (Springer, Berlin, 1996), p. 294.

[7] T. A. Duke and R. H. Austin, Phys. Rev. Lett. 80, 1552 (1998).
[8] D. Ertas, Phys. Rev. Lett. 80, 1548 (1998).

[9] I. Derényi and R. D. Astumian, Phys. Rev. E 58, 7781 (1998).

[10] A. van Oudenaarden and S. G. Boxer, Science 285, 1046 (1999).

[11] M. Kostur and L. Schimansky-Geier, Phys. Lett. A 265, 337 (2000).

[12] M. Bier, M. Kostur, I. Derényi, and R. D. Astumian, Phys. Rev. E 61, 7184 (2000).

[13] E. Anderson et al., LAPACK Users' Guide, 3rd ed. (SIAM, Philadelphia, 1999). 